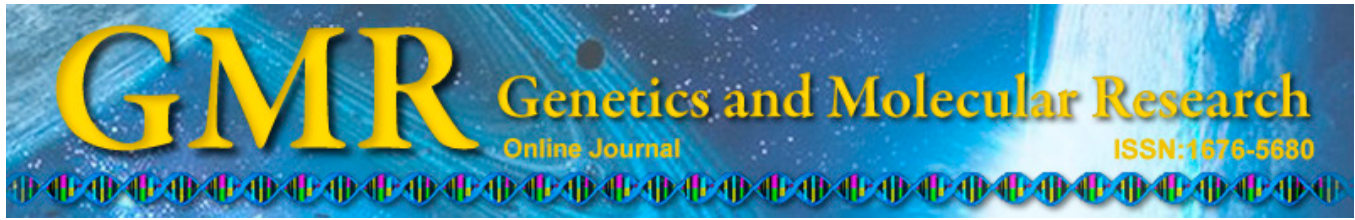

\title{
Effect of a pre-microRNA-149 (miR-149) genetic variation on the risk of ischemic stroke in a Chinese Han population
}

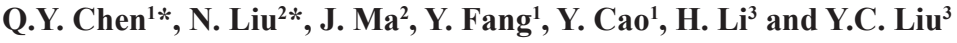 \\ ${ }^{1}$ Department of Central Laboratory, \\ The Affiliated People's Hospital of Jiangsu University, Zhenjiang, China \\ ${ }^{2}$ Department of Neurology, \\ The Affiliated People's Hospital of Jiangsu University, Zhenjiang, China \\ ${ }^{3}$ Department of Clinical Laboratory, The Taixing People's Hospital, \\ Taixing, China \\ *These authors contributed equally to this study. \\ Corresponding author: $\mathrm{H}$. Li \\ E-mail: realnow@sina.cn
}

Genet. Mol. Res. 14 (1): 2582-2589 (2015)

Received March 12, 2014

Accepted July 2, 2014

Published March 30, 2015

DOI http://dx.doi.org/10.4238/2015.March.30.17

\begin{abstract}
Clinical and experimental data have demonstrated that genetic factors play an important role in determining the susceptibility to ischemic stroke (IS). The present study was performed to clarify the association between the pre-microRNA-149 (miR-149) single nucleotide polymorphism rs71428439 and the risk of IS in the Jiangsu Han population. Polymerase chain reaction and restriction fragment length polymorphism were performed to identify the genotypes of the miR-149 single-nucleotide polymorphism rs71428439 in 730 unrelated subjects (IS, 348; healthy controls, 382). Plasma levels of homocysteine were determined using a radioassay kit. Compared to healthy controls, IS patients had a lower frequency of GG genotype distribution of the hsa-mir-149 polymorphism (11.5 vs 16.0\%) and a higher frequency of TT (46.6 vs 39.0\%). The risk of IS was significantly
\end{abstract}


lower among subjects carrying the GG genotype than subjects carrying the AA genotype (odds ratio (95\% confidence interval): 0.603 ( $0.382-$ $0.952), P=0.030$ ) or at least carrying the $G$ allele than patients carrying the A allele (odds ratio (95\% confidence interval): 0.769 (0.620-0.954), $\mathrm{P}=0.019$ ). Levels of folate were statistically higher in patients with the TT genotype $(8.59 \pm 7.75 \mathrm{ng} / \mathrm{mL})$ than in those with the CC genotype $(6.32 \pm 5.97 \mathrm{ng} / \mathrm{mL})$ in IS patients. Our results suggest that the miR149 single nucleotide polymorphism rs71428439 influences plasma levels of homocysteine and is associated with IS risk in the Jiangsu Han population.

Key words: Ischemic stroke; miR-149; Polymorphism

\section{INTRODUCTION}

Stroke is a multifactorial disorder with a high morbidity, mortality, disability, and recurrence rate in many developed countries (Hankey, 2006; Dichgans, 2007). There are 2 main types of stroke: ischemic strokes (IS) and hemorrhagic strokes. Hemorrhagic strokes account for $10-15 \%$ of all strokes. The remaining are IS, caused by a disruption of blood flow, oxygen, or glucose to the brain (Bendixen and Ocava, 2002). IS is the most commonly observed type of stroke in Chinese and accounts for the third major cause of morbidity and mortality in adults, behind only heart disease and cancer (Armstead et al., 2010). In China, IS is a leading cause of death and accounts for approximately two-thirds of all stroke-related deaths (Zhang et al., 2003). However, the pathophysiology of IS is complex and not completely understood. Data from family-based studies, twin studies, and animal experiments indicate that genetic factors play an important role in stroke (Jeffs et al., 1997; Liao et al., 1997; de Lange et al., 2001).

MicroRNAs (miRNAs) are a class of naturally occurring, small non-protein-coding RNAs of 21-24 nucleotides in length that regulate gene expression by base-pairing with target mRNAs at the 3'-untranslated region, leading to mRNA cleavage or translational repression (Lagos-Quintana et al., 2001; Lau et al., 2001). It has been suggested that miRNAs are important post-transcriptional regulators of gene expression and play a role in multiple cellular pathways. including cell proliferation, differentiation, cell cycle progression, and apoptosis (McDaneld, 2009). Accumulating evidence indicates that aberrant miRNA expression is involved in various diseases, including tumors, Down syndrome, schizophrenia, Alzheimer disease, Parkinson disease, and stroke (Jeon et al., 2013).

Recently, a well-known miRNA polymorphism in pre-miRNA sequences [miR-149 $\mathrm{T}>\mathrm{C}$ (rs2292832; chromosome 2, 241395503)] has been studied in a variety of diseases and was found to contribute to pathogenesis of these diseases (Chen et al., 2013; Zhang et al., 2013). Ding et al. (2013) found that a pre-miRNA-149 (pre-miR-149) genetic variation affects miR149 maturation. Bioinformatic analysis revealed that 5,10-methylenetetrahydrofolate reductase (MTHFR) may be a target gene of miR-149. A common polymorphism (C677T) in the gene encoding MTHFR, a critical enzyme in homocysteine metabolism, has been reported to be associated with both elevated plasma homocysteine levels and increased stroke risk in several studies (Frosst et al., 1995). However, there are currently no data regarding the role of miR-149 polymorphisms on homocysteine levels and stroke pathogenesis in a Chinese Han population. Therefore, we investigated the genetic association between the pre-miR-149 variant and IS. 


\section{MATERIAL AND METHODS}

\section{Study subjects}

A total of 348 patients with IS were enrolled from the Department of Neurology at The Affiliated People's Hospital, Jiangsu University. All study protocols were approved by The Institutional Review Board of The Affiliated People's Hospital, Jiangsu University, and written informed consent was obtained from each participant. The diagnosis of all stroke patients was confirmed to be IS using brain computed tomography or magnetic resonance imaging (Liao et al., 2013). Patients with history of myocardial infarction, cerebral vein thrombosis, transient ischemic attack, or hemorrhagic stroke were excluded. We selected 382 gender- and age ( \pm 5 years)-matched control subjects from patients presenting at our hospitals during the same period for health examinations. Control subjects had no recent history of cerebrovascular disease or myocardial infarction. Exclusion criteria were the same as those used for the patient group. All study participants were Chinese Han residing in Jiangsu. Subjects were deemed to have hypertension if the systolic blood pressure was $\geq 140 \mathrm{mmHg}$ or diastolic pressure was $\geq 90 \mathrm{mmHg}$, or if they were taking antihypertensive medications. Diabetes mellitus was defined as a fasting plasma glucose level $>126 \mathrm{mg} / \mathrm{dL}$ $(7.0 \mathrm{mM})$ and included patients taking diabetic medications. Smoking refers to patients who currently smoke. Hyperlipidemia was defined by total cholesterol serum levels $\geq 200 \mathrm{mg} / \mathrm{dL}$ or an antihyperlipidemic agent treatment history.

\section{Genotyping}

DNA was extracted from leukocytes using the Qiagen DNA Blood Mini kit (Qiagen, Hilden, Germany) according to manufacturer instructions. Genotypes of rs 71428439 were analyzed by polymerase chain reaction (PCR)-based direct DNA sequencing. DNA specimens were amplified by using standard PCR protocols. The primers were designed by Tu et al. (2012). The PCR profile included an initial melting step at $94^{\circ} \mathrm{C}$ for $5 \mathrm{~min}, 35$ cycles at $94^{\circ} \mathrm{C}$ for $60 \mathrm{~s}, 55^{\circ} \mathrm{C}$ for $45 \mathrm{~s}$, and $72^{\circ} \mathrm{C}$ for $1 \mathrm{~min}$, followed by a final extension step at $72^{\circ} \mathrm{C}$ for 10 min. The PCR product was a 493-bp fragment covering the A-G polymorphism site (rs71428439) in the miR-149 precursor.

\section{Laboratory analyses}

We collected plasma samples to measure total homocysteine levels within $48 \mathrm{~h}$ of stroke onset in patients. Additionally, 12-14 h after the subject's previous meal, $5 \mathrm{~mL}$ whole peripheral blood was collected in a tube containing anticoagulant. We centrifuged the tube for $10 \mathrm{~min}$ at $1000 \mathrm{~g}$ to separate the plasma. Plasma levels of homocysteine were determined using a radio-assay kit (Bio-Rad, Hercules, CA, USA), according to manufacturer instructions.

\section{Statistical analysis}

The SPSS 12.0 statistical software was applied for statistical analysis (SPSS, Inc., Chicago, IL, USA). Hardy-Weinberg equilibrium analysis was evaluated for the miR-149 alleles in the patient and control groups. The significance of differences between allelic and phe- 
notype frequencies in study groups was determined using the Pearson $\chi^{2}$ test. The relationship between genetic polymorphisms and IS was analyzed by the odds ratio (OR) and its $95 \%$ confidence interval $(95 \% \mathrm{CI})$, which was obtained by logistic regression. Statistical significance was accepted at the $\mathrm{P}<0.05$ level.

\section{RESULTS}

\section{Study population}

A total of 348 IS patients and 382 controls were included in the study. All patients belonged mainly to a Southeast Chinese Han population from Jiangsu. The clinical and demographic data for the study subjects, including age, gender, hypertension, smoking status, diabetes mellitus, hyperlipidemia, smoking, and homocysteine, are shown in Table 1 . The mean age was $66.3 \pm 10.2$ years in the control group and $65.7 \pm 11.5$ years in the patient group. No statistical difference was observed in age or gender distribution between the control and patient groups. However, a significantly higher proportion of IS patients were smokers compared to the control group $(\mathrm{P}<0.05)$. IS patients were significantly more likely to have high-density lipoprotein cholesterol, diabetes mellitus, hypertension, hyperlipidemia, and increased homocysteine levels $(\mathrm{P}<0.05)$.

Table 1. Baseline characteristics of ischemic stroke patients.
\begin{tabular}{lccc}
\hline Characteristics & Control & Ischemic stroke & P value \\
\hline Total (N) & 348 & 382 & $0.399^{*}$ \\
Male [N (\%)] & 193 & 205 & $0.455^{*}$ \\
Age, years (mean $\pm \mathrm{SD})$ & $66.3 \pm 10.2$ & $65.7 \pm 11.5$ & $0.005^{*}$ \\
Hypertension [N (\%)] & $136(39.1)$ & $190(49.7)$ & $0.010^{*}$ \\
Diabetes mellitus [N (\%)] & $45(12.9)$ & $77(17.5)$ & $0.036^{*}$ \\
Hyperlipidemia [N (\%)] & $69(19.8)$ & $101(26.4)$ & $0.010^{*}$ \\
Smoking [N (\%)] & $92(26.4)$ & $135(35.3)$ & $0.000^{*}$ \\
Homocysteine (ng/mL) & $10.12 \pm 5.19$ & $12.43 \pm 6.09$ & \\
\hline
\end{tabular}

Compared to controls $* \mathrm{P}<0.05,{ }^{*} \mathrm{P}>0.05$.

\section{Association between hsa-mir-149 polymorphism and IS}

The genotype at rs71428439 was identified in patients and healthy volunteers. The allele and genotype distributions are shown in Table 2. The distribution of genotypes for the pre-miR-149 SNP rs71428439 in the patient and healthy volunteer groups complied with Hardy-Weinberg equilibrium (patient group: $\mathrm{P}=0.419$; healthy volunteers: $\mathrm{P}=$ 0.338). The AG genotype of the hsa-mir-149 gene was more common in the control group, while the AA genotype of pre-miR-149 gene was more common in the patient group. Compared to healthy controls, IS patients had a lower frequency of the GG genotype of the hsa-mir-149 polymorphism (11.5 vs 16.0\%) and a higher frequency of TT (46.6 vs $39.0 \%$ ). The risk of IS was significantly lower among subjects carrying the GG genotype than in subjects carrying the AA genotype (OR (95\%CI): $0.603(0.382-0.952), \mathrm{P}=0.030)$ or at least carrying the $\mathrm{G}$ allele than patients carrying the A allele (OR $(95 \% \mathrm{CI}): 0.769$ (0.620-0.954), $\mathrm{P}=0.019)$. 
Table 2. Distribution of miR-149 genotypes in patients and the control group.

\begin{tabular}{llcccc}
\hline & & Control $[\mathrm{N}(\%)]$ & Ischemic stroke $[\mathrm{N}(\%)]$ & OR $(95 \% \mathrm{CI})$ & $\mathrm{P}$ \\
\hline rs71428439 & AA & $149(39.0)$ & $162(46.6)$ & & \\
& AG & $172(45.0)$ & $146(42.0)$ & $0.781(0.571-1.068)$ & 0.130 \\
& GG & $61(16.0)$ & $40(11.5)$ & $0.603(0.382-0.952)$ & 0.030 \\
& A allele & 470 & 470 & $0.769(0.620-0.954)$ & 0.019 \\
\hline
\end{tabular}

\section{Effect of hsa-mir-149 polymorphism on plasma levels of homocysteine}

Bioinformatic analysis revealed that MTHFR is a target gene of miR-149 (Figure 1A). We assessed the plasma levels of homocysteine in patients with different genotypes. As shown in Figure 1, homocysteine levels were statistically lower in patients with the GG or AG genotype $(11.53 \pm 5.02$ or $12.31 \pm 5.29 \mathrm{ng} / \mathrm{mL})$ than in those with the AG or AA genotype (15.37 $\pm 7.31 \mathrm{ng} / \mathrm{mL}$ ) in IS patients (Figure 1B). No statistical difference was observed in the plasma levels of homocysteine between the AG and $\mathrm{GG}$ genotypes $(\mathrm{P}=0.3895)$.

\section{A Position 342-348 of MTHFR 3' UTR}

$$
\text { hsa-miR-149 }
$$

\section{5. - ...CCAGGGCAGCCUCCAGAGCCAGC... 3. CCCUCACUUCUgUGCCUCGgUCU}

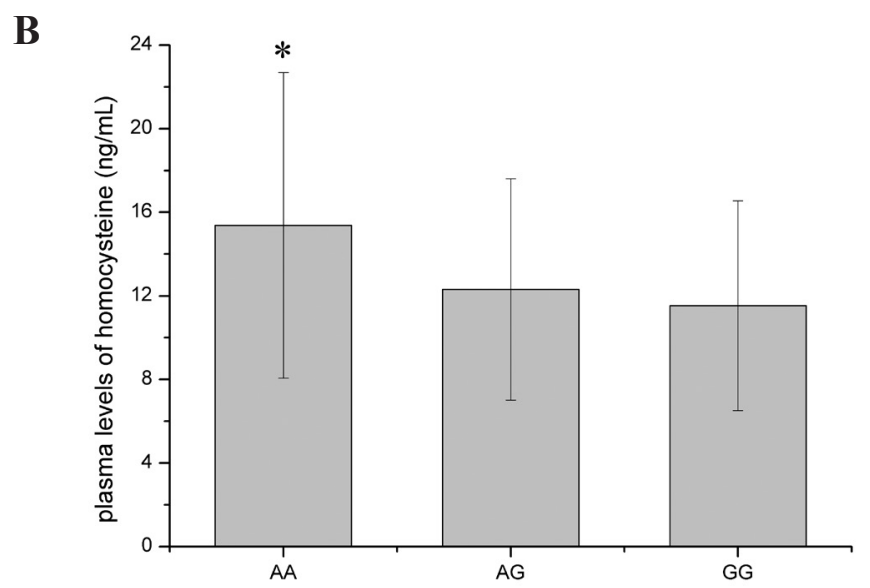

Figure 1. Effect of pre-miR-149 polymorphism rs71428439 on plasma levels of homocysteine. A. Targetscan predicted that MTHFR was a target gene of miR-149. B. Plasma levels of homocysteine were statistically lower in patients with the GG genotype compared to the AA genotype group, ${ }^{*} \mathrm{P}<0.05$. 


\section{DISCUSSION}

In the present study, based on previous reports, we selected a potential functional SNP rs71428439 in miR-149 and investigated its relationship with IS in a Chinese Han population. We found that rs71428439 may affect the susceptibility to IS. This is the first study to evaluate the association between rs71428439 polymorphisms and IS in a Chinese Han population. Our findings suggest that genetic variants of pre-miR-149 play a role in IS pathogenesis.

Stroke is a complex disease caused by a combination of various genetic and environmental factors and is the leading cause of long-term disability and mortality in China. Its incidence is more than 4-5 times higher than that in developed countries (Wu et al., 2012). miRNAs are known to play important roles in many physiological and pathological processes, including tumorigenesis (Mocellin et al., 2009), proliferation (Johnnidis et al., 2008), hematopoiesis (Merkerova et al., 2008), metabolism (Aumiller and Förstemann, 2008), immune function (Carissimi et al., 2009), epigenetics, and neurodegenerative diseases (Bushati and Cohen, 2008). miRNAs have also been found to be beneficial for identifying the etiology of lymphoma (Lawrie et al., 2007) and progression of certain neurological diseases (Nelson et al., 2008). Over the past decade, miRNA microarray has been performed to examine the differential expression profiles of miRNAs in stroke in vivo and in vitro. The studies indicated that miRNAs are key modulators and play important roles in both pathogenic and pathological aspects of IS biology.

Jeyaseelan et al. (2008) determined an miRNA profile under ischemic conditions from the ischemic brain and blood of rats suffering middle cerebral artery occlusion and found that some of the miRNAs that are overexpressed in the ischemic brain tissue can be detected in serum or plasma. An miRNA expression profile has been implicated in endothelial/vascular function, erythropoiesis, angiogenesis, and neural function, demonstrating the potential of using peripheral blood miRNA profiles as biomarkers of cerebral IS (Kong et al., 2011). Similarly to cardiac-specific miRNA, Kawamoto et al. (2005) found that miR-124 was a brainspecific microRNA and was increased in the plasma of a rat acute IS model, generating interest in the exploration of brain-specific miRNAs, including miR-124, miR-134, miR-153, miR-9, and miR-219, as potential molecular biomarkers of human acute IS (Laterza et al., 2009). However, few studies have examined the roles of genetic variation in IS. In this study, we investigated the association between the pre-miR-149 (rs71428439) polymorphism and IS.

MTHFR is a key enzyme involved in folate metabolism. It catalyzes the conversion of 5,10-methylenetetrahydrofolate to 5-methyltetrahydrofolate. This may be used as a predictive marker for IS in patients with associated type 2 diabetes (Kawamoto et al., 2005). The effects of MTHFR C677T polymorphism appear to be more common in regions with low dietary folate consumption than in those with high dietary folate consumption (Holmes et al., 2011). However, some recent studies observed no direct association between any single polymorphism of key genes of the homocysteine metabolic pathway (Low et al., 2011). In the present study, we found that the risk of IS was significantly lower among subjects carrying the GG genotypes than subjects carrying the AA genotype or at least carrying one G allele than among patients carrying the A allele. To investigate the gene-environment interaction, we explored the combined effects of pre-miR-149 polymorphisms and plasma tHcy levels. IS patients show increased tHcy levels compared to controls $(\mathrm{P}<0.05)$. Bioinformatic analysis revealed that MTHFR is a target gene of miR-149. The polymorphism in pre-miR-149 may modulate folate levels by modulating MTHFR activity. 
In summary, we found that the miR-149 polymorphism is correlated with susceptibility to IS in a Chinese Han population. These findings add to the body of evidence demonstrating a role for microRNA in disease susceptibility and pathology. Additional studies should be conducted to provide a detailed understanding of the molecular mechanisms linking the miR149 polymorphism to IS.

\section{REFERENCES}

Armstead WM, Kiessling JW, Kofke WA and Vavilala MS (2010). SNP improves cerebral hemodynamics during normotension but fails to prevent sex dependent impaired cerebral autoregulation during hypotension after brain injury. Brain Res. 1330: 142-150.

Aumiller V and Förstemann K (2008). Roles of microRNAs beyond development - metabolism and neural plasticity. Biochim. Biophys. Acta 1779: 692-696.

Bendixen BH and Ocava L (2002). Evaluation and management of acute ischemic stroke. Curr. Cardiol. Rep. 4: 149-157.

Bushati N and Cohen SM (2008). MicroRNAs in neurodegeneration. Curr. Opin. Neurobiol. 18: 292-296.

Carissimi C, Fulci V and Macino G (2009). MicroRNAs: novel regulators of immunity. Autoimmun. Rev. 8: 520-524.

Chen Z, Xu L, Ye X, Shen S, et al. (2013). Polymorphisms of microRNA sequences or binding sites and lung cancer: a meta-analysis and systematic review. PLoS One 8: e61008.

de Lange M, Snieder H, Ariëns RA, Spector TD, et al. (2001). The genetics of haemostasis: a twin study. Lancet 357 : 101-105.

Dichgans M (2007). Genetics of ischaemic stroke. Lancet Neurol. 6: 149-161.

Ding SL, Wang JX, Jiao JQ, Tu X, et al. (2013). A pre-microRNA-149 (miR-149) genetic variation affects miR-149 maturation and its ability to regulate the Puma protein in apoptosis. J. Biol. Chem. 288: 26865-26877.

Frosst P, Blom HJ, Milos R, Goyette P, et al. (1995). A candidate genetic risk factor for vascular disease: a common mutation in methylenetetrahydrofolate reductase. Nat. Genet. 10: 111-113.

Hankey GJ (2006). Potential new risk factors for ischemic stroke: what is their potential? Stroke 37: 2181-2188.

Holmes MV, Newcombe P, Hubacek JA, Sofat R, et al. (2011). Effect modification by population dietary folate on the association between MTHFR genotype, homocysteine, and stroke risk: a meta-analysis of genetic studies and randomised trials. Lancet 378: 584-594.

Jeffs B, Clark JS, Anderson NH, Gratton J, et al. (1997). Sensitivity to cerebral ischaemic insult in a rat model of stroke is determined by a single genetic locus. Nat. Genet. 16: 364-367.

Jeon YJ, Kim OJ, Kim SY, Oh SH, et al. (2013). Association of the miR-146a, miR-149, miR-196a2, and miR-499 polymorphisms with ischemic stroke and silent brain infarction risk. Arterioscler. Thromb. Vasc. Biol. 33: 420-430.

Jeyaseelan K, Lim KY and Armugam A (2008). MicroRNA expression in the blood and brain of rats subjected to transient focal ischemia by middle cerebral artery occlusion. Stroke 39: 959-966.

Johnnidis JB, Harris MH, Wheeler RT, Stehling-Sun S, et al. (2008). Regulation of progenitor cell proliferation and granulocyte function by microRNA-223. Nature 451: 1125-1129.

Kawamoto R, Kohara K, Oka Y, Tomita H, et al. (2005). An association of 5,10-methylenetetrahydrofolate reductase (MTHFR) gene polymorphism and ischemic stroke. J. Stroke Cerebrovasc. Dis. 14: 67-74.

Kong L, Zhu J, Han W, Jiang X, et al. (2011). Significance of serum microRNAs in pre-diabetes and newly diagnosed type 2 diabetes: a clinical study. Acta Diabetol. 48: 61-69.

Lagos-Quintana M, Rauhut R, Lendeckel W and Tuschl T (2001). Identification of novel genes coding for small expressed RNAs. Science 294: 853-858.

Laterza OF, Lim L, Garrett-Engele PW, Vlasakova K, et al. (2009). Plasma MicroRNAs as sensitive and specific biomarkers of tissue injury. Clin. Chem. 55: 1977-1983.

Lau NC, Lim LP, Weinstein EG and Bartel DP (2001). An abundant class of tiny RNAs with probable regulatory roles in Caenorhabditis elegans. Science 294: 858-862.

Lawrie CH, Soneji S, Marafioti T, Cooper CD, et al. (2007). MicroRNA expression distinguishes between germinal center B cell-like and activated B cell-like subtypes of diffuse large B cell lymphoma. Int. J. Cancer 121: 1156-1161.

Liao D, Myers R, Hunt S, Shahar E, et al. (1997). Familial history of stroke and stroke risk. The Family Heart Study. Stroke 28: 1908-1912.

Liao YC, Lin HF, Guo YC, Chen CH, et al. (2013). Lack of association between a functional variant of the BRCA-1 related associated protein (BRAP) gene and ischemic stroke. BMC Med. Genet. 14: 17.

Low HQ, Chen CP, Kasiman K, Thalamuthu A, et al. (2011). A comprehensive association analysis of homocysteine 
metabolic pathway genes in Singaporean Chinese with ischemic stroke. PLoS One 6: e24757.

McDaneld TG (2009). MicroRNA: mechanism of gene regulation and application to livestock. J. Anim. Sci. 87: E21-E28.

Merkerova M, Belickova M and Bruchova H (2008). Differential expression of microRNAs in hematopoietic cell lineages. Eur. J. Haematol. 81: 304-310.

Mocellin S, Pasquali S and Pilati P (2009). Oncomirs: from tumor biology to molecularly targeted anticancer strategies. Mini Rev. Med. Chem. 9: 70-80.

Nelson PT, Wang WX and Rajeev BW (2008). MicroRNAs (miRNAs) in neurodegenerative diseases. Brain Pathol. 18: 130-138.

Tu HF, Liu CJ, Chang CL, Wang PW, et al. (2012). The association between genetic polymorphism and the processing efficiency of miR-149 affects the prognosis of patients with head and neck squamous cell carcinoma. PLoS One 7: e51606.

Wu P, Zuo X and Ji A (2012). Stroke-induced microRNAs: The potential therapeutic role for stroke. Exp. Ther. Med. 3: 571-576.

Zhang LF, Yang J, Hong Z, Yuan GG, et al. (2003). Proportion of different subtypes of stroke in China. Stroke 34: 20912096.

Zhang YG, Shi JX, Song CH, Wang P, et al. (2013). Association of mir-499 and mir-149 Polymorphisms with Cancer Risk in the Chinese Population: Evidence from Published Studies. Asian Pac. J. Cancer Prev. 14: 2337-2342. 